\title{
TB immunodiagnosis in context of global disease care and control
}

\author{
Mark D Perkins \\ From Immunodiagnosis of Tuberculosis: New Questions, New Tools \\ Virginia, VA, USA. 21-23 September 2008
}

The persistence of the tuberculosis epidemic depends upon ongoing transmission from undetected and untreated cases. Unfortunately, though high cure rates have now been achieved in many countries, globally case detection is delayed and incomplete, both because diagnostic testing is relatively hard to access, and because it relies on inadequate technologies. Fewer than half of all TB cases are thought to be detectable by microscopy even if all suspects were appropriately examined. Delays in diagnosis are common, and patients are often ill for 4 to 8 months before they are detected and treated. During this delay, morbidity accrues and transmission to household and community contacts continues.

To improve TB case detection, new technologies are needed which are more rapid than culture and that are either highly sensitive, such as new-generation molecular tests, or simple enough to use that they can be widely available, even if imperfectly sensitive. Lateral flow immunoassays are among the simplest clinical diagnostic tests to perform, and are routinely used outside laboratory settings to detect conditions such as pregnancy and malaria. The availability of a well-performing seroimmunodiagnostic test for tuberculosis on a lateral flow platform could vastly simplify case detection and improve TB care and control if testing. Such assays are relatively simple to develop, and in fact more than two dozen such tests are marketed in the private sector, primarily in Asia. Unfortunately, of the many commercially-manufactured tests currently available to detect anti-TB antibody responses, none are accurate enough to be recommended for use in disease control programs. A limited number of TB antigens, however, have been explored for their diagnostic potential, and the on-going discovery of new antigens may allow development of improved serodiagnostic tests. To this end, FIND is collaborating with a team of scientists to interrogate the entire TB proteome to identify antigen candidates for new tests.

In collaboration with Felgner and colleagues (Antigen Discovery, Inc.), microarrays containing the entire TB proteome have been created using a high-throughput cloning and expression system. At PHRI, Gennaro and colleagues have used these arrays to screen serum samples from TB patients with and without concomitant HIV infection, and appropriate controls. A subset of the most promising antigens will be taken forward for development of an optimized seroimmunodiagnostic. The goal is a test that is either sensitive enough to exclude TB in test-negative symptomatic individuals, or specific enough to direct therapy in those who are positive.

Published: 17 December 2010

doi:10.1186/1753-6561-4-S3-O16

Cite this article as: Perkins: TB immunodiagnosis in context of global

disease care and control. BMC Proceedings 2010 4(Suppl 3):016. 